Article

\title{
Evaluating the Effect of Varying the Metal Precursor in the Colloidal Synthesis of MoSe 2 Nanomaterials and Their Application as Electrodes in the Hydrogen Evolution Reaction
}

\author{
Zakhele Ndala $^{1}$, Ndivhuwo Shumbula ${ }^{1}{ }^{10}$, Siyabonga Nkabinde ${ }^{1}$, Tshwarela Kolokoto ${ }^{1}$, \\ Obakeng Nchoe ${ }^{1}$, Poslet Shumbula ${ }^{2}$, Zikhona N. Tetana 1,3,4, Ella C. Linganiso 1,3,4, \\ Siziwe S. Gqoba ${ }^{1, *}$ and Nosipho Moloto ${ }^{1, * \mathbb{D}}$
}

1 Molecular Sciences Institute, School of Chemistry, University of the Witwatersrand, Private Bag 3, Wits 2050, South Africa; 491384@students.wits.ac.za (Z.N.); 677753@students.wits.ac.za (N.S.); 564058@students.wits.ac.za (S.N.); 670850@students.wits.ac.za (T.K.); 2291563@students.wits.ac.za (O.N.); Zikhona.Tetana@wits.ac.za (Z.N.T.); Cebisa.Linganiso@wits.ac.za (E.C.L.)

2 Department of Chemistry, University of Limpopo Private Bag x1106, Sovenga 0727, South Africa; poslet.shumbula@ul.ac.za

3 DST/NRF Centre of Excellence in Strong Materials, University of the Witwatersrand, Private Bag 3, Wits 2050, South Africa

4 Microscopy and Microanalysis Unit, University of the Witwatersrand, Private Bag 3, Johannesburg, Wits 2050, South Africa

* Correspondence: Siziwe.Gqoba@wits.ac.za (S.S.G.); Nosipho.Moloto@wits.ac.za (N.M.); Tel.: +27-11-7176-774 or +27-11-7176-756 (S.S.G.); Fax: +27-11-7176-749 (N.M.)

Received: 7 July 2020; Accepted: 24 July 2020; Published: 9 September 2020

\begin{abstract}
Herein we report on the use of different metal precursors in the synthesis of $\mathrm{MoSe}_{2}$ nanomaterials in order to control their morphology. The use of $\mathrm{Mo}(\mathrm{CO})_{6}$ as the metal precursor resulted in the formation of wrinkled few-layer nanosheets, while the use of $\mathrm{H}_{2} \mathrm{MoO}_{4}$ as the metal precursor resulted in the formation of nanoflowers. To investigate the effect of the morphologies on their performance as catalysts in the hydrogen evolution reaction, electrochemical characterization was done using linear sweep voltammetry (LSV), cyclic voltammetry (CV), and electrical impedance spectroscopy (EIS). The $\mathrm{MoSe}_{2}$ nanoflowers were found to have superior electrochemical performance towards the hydrogen evolution reaction with a lower Tafel slope, on-set potential, and overpotential at $10 \mathrm{~mA} / \mathrm{cm}^{2}$ compared to the wrinkled few-layer nanosheets. This was found to be due to the higher effective electrochemical surface area of the nanoflowers compared to the nanosheets which suggests a higher number of exposed edge sites in the nanoflowers.
\end{abstract}

Keywords: molybdenum diselenide; colloidal synthesis; HER

\section{Introduction}

2D layered materials such as $\mathrm{MoSe}_{2}$ are quickly becoming some of the most exciting materials, with interesting properties that have put them at the forefront for use in various applications and technologies including energy storage and generation devices, field-effect transistors and biosensors [1-3]. $\mathrm{MoSe}_{2}$ is a layered transition metal dichalcogenide (TMD) material where each layer is comprised of atom-thin layers of transition metal sandwiched between two chalcogen layers. Monolayer and few-layer $\mathrm{MoSe}_{2}$ nanomaterials have been shown to have interesting properties that can be used in various applications [4-6]. Colloidal synthesis is a wet-chemical technique that has been identified to have the potential to synthesize TMDs such as $\mathrm{MoSe}_{2}$ in high yields with control 
over the size, morphology and layer thickness which would, in turn, control their properties [7-9]. This control is achieved through the modification of the reaction parameters such as the temperature, chemical reagents, solvent/surfactant, and reagent concentration.

Unfortunately, achieving this level of control on the properties of TMD nanomaterials is not a small task and is currently a significant challenge in the synthesis of TMDs using colloidal synthetic techniques. There has been a significant effort to investigate how the reaction parameters can be used to control some properties of the TMD nanomaterials [10-12]. Much of the work on the effect of precursors on the synthesis of $\mathrm{MoSe}_{2}$ nanomaterials has focused on the effect of the selenium precursor and largely ignored is the effect of the metal precursor [13-15]. The effect of the metal precursor choice in colloidal syntheses has been shown to be crucial in controlling the size, morphology, and phase of nanomaterials [16-19]. This work investigates the unexplored effect of two different metal precursors on the morphology of $\mathrm{MoSe}_{2}$ nanomaterials. In this work molybdenum hexacarbonyl and molybdic acid were used to evaluate the effect of these different metal precursors on the morphology of $\mathrm{MoSe}_{2}$ nanomaterials.

To demonstrate how important a difference in the morphology of the $\mathrm{MoSe}_{2}$ nanomaterials can have on any application, the electrochemical properties of the nanomaterials were evaluated for application of the nanomaterials in the hydrogen evolution reaction (HER). The hydrogen evolution reaction is an electrocatalytic technique that is used in the production of hydrogen gas. This process is important because it provides a way to produce hydrogen which is a clean and efficient energy carrier in an eco-friendly manner [20]. $\mathrm{MoSe}_{2}$ has been identified as a potentially cost-effective alternative to platinum which is the current catalyst used for the HER [21,22]. The active sites for the HER in $\mathrm{MoSe}_{2}$ have been identified as the edge sites [22,23]. Thus, finding a morphology that maximizes the exposure of these edge sites in $\mathrm{MoSe}_{2}$ is crucial. This work shows how changing the morphology of the $\mathrm{MoSe}_{2}$ nanomaterials using the metal precursors influences the catalytic activity of the nanomaterials.

\section{Experimental}

\subsection{Chemicals}

Molybdenum hexacarbonyl ( $\mathrm{Mo}(\mathrm{CO})_{6}, 98 \%$, Sigma-Aldrich, Modderfontein, South Africa), molybdic acid $\left(\mathrm{H}_{2} \mathrm{MoO}_{4}, 85 \%\right.$, Sigma-Aldrich), selenourea $\left(\mathrm{CH}_{4} \mathrm{~N}_{2} \mathrm{Se}, 98 \%\right.$, Sigma-Aldrich), oleylamine (OAm, 70\%, Sigma-Aldrich), toluene (anhydrous, 99.8\%, Sigma-Aldrich) and ethanol (absolute, $\geq 99.8 \%$, Sigma-Aldrich) were used as received without further purification.

\subsection{Synthesis of $\mathrm{MoSe}_{2}$ Nanomaterials}

\subsubsection{Synthesis of $\mathrm{MoSe}_{2}$ Wrinkled Few-Layer Nanosheets}

A three-neck round bottom flask was filled with $20 \mathrm{~mL}$ of OAm and degassed in $\mathrm{N}_{2}$ for $20 \mathrm{~min}$. The temperature was increased to $50^{\circ} \mathrm{C}$ and $0.362 \mathrm{~g}(2.49 \mathrm{mmol})$ of selenourea was added to the reaction mixture and stirred for $10 \mathrm{~min}$. At $220^{\circ} \mathrm{C}$, the selenourea decomposes to $\mathrm{H}_{2} \mathrm{Se}$ and a carboamide $\left(\mathrm{C}(\mathrm{NH})_{2}\right)$, this is shown in the TGA profile in Figure S1. After selenourea decomposes the colour of the mixture changed from black to a dark orange colour. Molybdenum hexacarbonyl $(0.212 \mathrm{~g}, 0.802 \mathrm{mmol})$ ) was dissolved in the OAm. The mixture of molybdenum hexacarbonyl and OAm was then injected into the selenourea mixture at $300^{\circ} \mathrm{C}$. The reaction mixture turned black immediately. The reaction was allowed to run for $120 \mathrm{~min}$. The black powder product was washed and collected by centrifugation using toluene and ethanol.

\subsubsection{Synthesis of $\mathrm{MoSe}_{2}$ Nanoflowers}

In a three-neck round bottom flask $20 \mathrm{~mL}$ of OAm was added, the solvent was degassed for $20 \mathrm{~min}$ under nitrogen. The temperature was increased to $50{ }^{\circ} \mathrm{C}$ and $0.4 \mathrm{~g}(3.0 \mathrm{mmol})$ of selenourea was added to the reaction mixture and stirred for $10 \mathrm{~min}$. About $0.2 \mathrm{~g}(0.15 \mathrm{mmol})$ of molybdic acid 
was added once the reaction temperature reached $300^{\circ} \mathrm{C}$. The reaction was allowed to run for $120 \mathrm{~min}$, after which the product was collected and washed with toluene and ethanol by centrifugation.

\subsection{Characterization Techniques}

The phase purity, crystallinity and preferred crystal orientation of the products were examined by using PXRD on a MeasSrv (D2-205530)/D2-205530 diffractometer (Bruker, Billerica, MA, USA) using secondary graphite monochromated $\operatorname{CoK} \alpha$ radiation $(\lambda 1.78897 \AA)$ at $30 \mathrm{kV} / 30 \mathrm{~mA}$. Measurements were taken using a glancing angle of incidence detector at an angle of $2^{\circ}$, for $2 \theta$ values over $10-90^{\circ}$ in steps of $0.026^{\circ}$ with a step time of $37 \mathrm{~s}$ and at a temperature of $25^{\circ} \mathrm{C}$. Raman spectroscopy experiments were performed on a J-Y T64000 micro-Raman spectrometer (Horiba Jobin-Yvon, Ltd., Stanmore, UK) equipped with a liquid nitrogen cooled charge-coupled device detector. All samples were measured after excitation with a laser wavelength of $514.5 \mathrm{~nm}$. The particle sizes and morphologies were determined by the transmission electron microscopy (TEM) carried out on a Technai T12 TEM microscope (FEI, Hillsboro, OR, USA) operated at an acceleration voltage of $200 \mathrm{kV}$ with a beam spot size of 20-100 nm in TEM mode. The HRTEM images were obtained from a JEM-2100 microscope (JEOL, Akishima, Tokyo, Japan) equipped with a LAB6 filament and an EDS detector, operated at 200 kV. SEM images were obtained with a high-resolution FEI Nova Nanolab 600 instrument at $30 \mathrm{kV}$ (FEI, Hillsboro, OR, USA). The total surface area and pore volume of the nanomaterials were ascertained using a Micromeritics TriStar Surface Area and Porosity Analyzer (Micromeritics Instrument Corp., Norcross, GA, USA). The electrochemical measurements were carried out on a BASi epsilon E2 (1231) (West Lafayette, IN, USA). All measurements were carried out in $0.5 \mathrm{M} \mathrm{H}_{2} \mathrm{SO}_{4}$ using a three-electrode system. A Ag/AgCl electrode was used as the reference electrode, platinum wire was used as the counter electrode. A modified glassy carbon electrode with $3 \mathrm{~mm}$ diameter was used as the working electrode. The ink or fresh dispersion of the sample was prepared by dispersing $5 \mathrm{mg}$ of the $\mathrm{MoSe}_{2}$ nanomaterials with $0.5 \mathrm{mg}$ carbon black and $40 \mu \mathrm{L}$ of Nafion solution ( $5 \mathrm{wt} \%$ ) in a $1 \mathrm{~mL}$ mixture of water and isopropanol at a 3:1 ratio. The solution was then sonicated for $30 \mathrm{~min}$ and $5 \mu \mathrm{L}$ of the ink was drop-casted onto the glassy carbon electrode. The electrode was allowed to dry at room temperature. The linear sweep voltammograms were obtained at a scan rate of $2 \mathrm{mV} / \mathrm{s}$ and the cyclic voltammograms were obtained at a potential range of $0.2-0.6 \mathrm{~V}$ vs RHE. A $20 \% \mathrm{Pt} / \mathrm{C}$ catalyst was used for comparison. The impedance spectroscopy studies were conducted on a SP 300 system (Biologic, Seyssinet-Pariset, France). The measurements were done at a potential of $-200 \mathrm{mV}$ vs RHE and it was done at a frequency range between $0.01 \mathrm{~Hz}$ and $100 \mathrm{kHz}$.

\section{Results and Discussion}

\subsection{Structural Characterisation}

Powder X-ray diffraction (PXRD) was used to confirm the synthesis of $\mathrm{MoSe}_{2}$ and to also determine the crystallinity, phase and composition of the nanomaterials. The PXRD confirms the formation of $2 \mathrm{H}$ hexagonal $\mathrm{MoSe}_{2}$ nanomaterials (JCPDS card no: 03-065-3999). The reflection peaks located at $14.63^{\circ}$, $37.06^{\circ}, 44.44^{\circ}$, and $66.02^{\circ}$, corresponding to the lattice planes (002), (100), (103), and (110) respectively. The broad nature of the peaks as shown in Figure 1 is indicative of nanostructured materials.

There are no peaks not belonging to $\mathrm{MoSe}_{2}$ in the diffractograms, thus we can state that the nanomaterials are free of any impurities. The (002) peak arises due to the stacking of the layers in multilayer nanosheets. The appearance of the (002) peak in both diffraction patterns suggests the formation of multilayer $\mathrm{MoSe}_{2}$ [18]. However, the (002) diffraction peak in the $\mathrm{MoSe}_{2}$ nanosheets seems much broader than the (002) diffraction peak of the $\mathrm{MoSe}_{2}$ nanoflowers. This broadening is attributed to an inhomogeneity in the spacing between the van der Waals planes [24]. The Raman spectrum of $\mathrm{MoSe}_{2}$ has two main characteristic peaks which are the out of plane $\mathrm{A}_{1 \mathrm{~g}}$ and the in plane $\mathrm{E}^{1}{ }_{2 \mathrm{~g}}$, the mono or few-layer nanomaterials are identified by the shift in position of these characteristic 
peaks from their positions in the bulk material [25]. The Raman spectra of $\mathrm{MoSe}_{2}$ nanosheets and $\mathrm{MoSe}_{2}$ nanoflowers are shown in Figure 2.

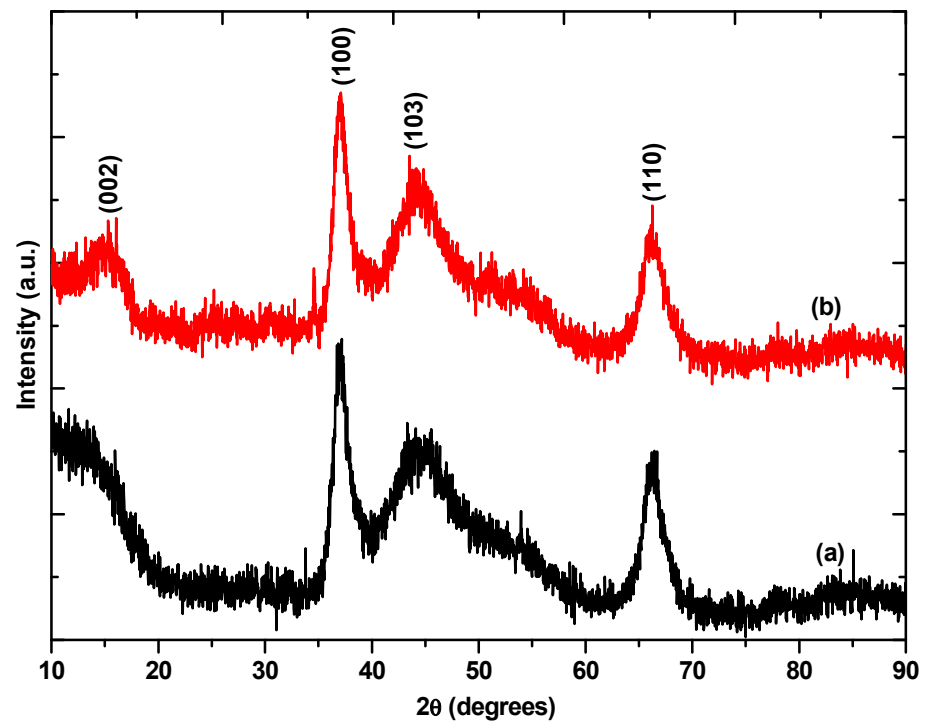

Figure 1. Powder X-ray diffraction patterns of (a) $\mathrm{MoSe}_{2}$ nanosheets (b) $\mathrm{MoSe}_{2}$ nanoflowers.

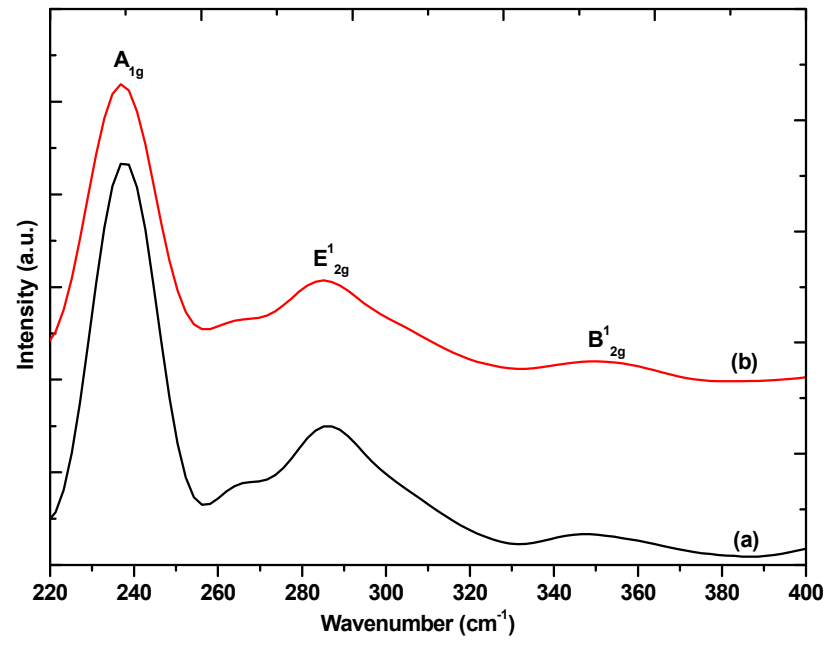

Figure 2. Raman spectra of (a) $\mathrm{MoSe}_{2}$ nanosheets and (b) $\mathrm{MoSe}_{2}$ nanoflowers.

The appearance of the characteristic out of plane $\mathrm{A}_{1 \mathrm{~g}}$ and in plane $\mathrm{E}^{1}{ }_{2 \mathrm{~g}}$ bands in both the Raman spectra confirms the formation of $\mathrm{MoSe}_{2}$. The $\mathrm{A}_{1 \mathrm{~g}}$ appears at $242 \mathrm{~cm}^{-1}$ and the $\mathrm{E}_{2 \mathrm{~g}}^{1}$ appears at $286 \mathrm{~cm}^{-1}$ in the bulk [25]. The Raman spectra for both the nanosheets and nanoflowers show the $\mathrm{A}_{1 \mathrm{~g}}$ has red-shifted from the bulk to $237 \mathrm{~cm}^{-1}$. The $\mathrm{E}^{1}{ }_{2 \mathrm{~g}}$ has shifted to $285 \mathrm{~cm}^{-1}$. The red-shift of both the $A_{1 g}$ and $E^{1}{ }_{2 g}$ is indicative of the formation of few-layers [26]. There is one other characteristic peak observed in both the Raman spectra, the $\mathrm{B}^{1}{ }_{2 \mathrm{~g}}$ which has been shown to not appear in monolayers or in the bulk materials. This peak only appears for few-layer materials with a layer number between 2-5 layers [25]. This result is in agreement with the PXRD analysis which confirmed the formation of few-layers nanosheets by the appearance of the (002) diffraction peak. The peak positions of the two samples do not show a significant difference from one another so the number of layers in the two samples is comparable.

The images in Figure 3a,c show a network of interconnected wrinkled few-layer nanosheets, while the images in Figure 3b,d show the formation of $\mathrm{MoSe}_{2}$ nanoflowers which are composed of small lateral dimension nanosheets that seem to form from one central point. To investigate the 
formation of the wrinkled few-layer nanosheets a time study of the reaction was conducted where aliquots were obtained at the 30,60, 90, and $120 \mathrm{~min}$ time intervals. Initial observation of the $30 \mathrm{~min}$ sample under low-resolution TEM revealed the appearance of a flocculate. This flocculate has also been observed by Savjani et al. in the synthesis of $\mathrm{MoSe}_{2}$. The flocculates have lateral dimensions of several hundred nanometres. They seem to mould and blanket the lacey carbon grids as shown in Figure 4a [27]. A closer look at these flocculates at higher magnification revealed that some ultrathin $\mathrm{MoSe}_{2}$ nanosheets had begun to form Figure $4 \mathrm{~b}$. These nanosheets seem to overlap with one another forming an interconnected network of few-layer nanosheets Figure 4c. The HRTEM images in Figure S2 confirms these as $\mathrm{MoSe}_{2}$ nanosheets due to the lattice fringes being $\sim 0.66 \mathrm{~nm}$ which is the d-spacing of the (002) lattice plane in multi-layer nanosheets.

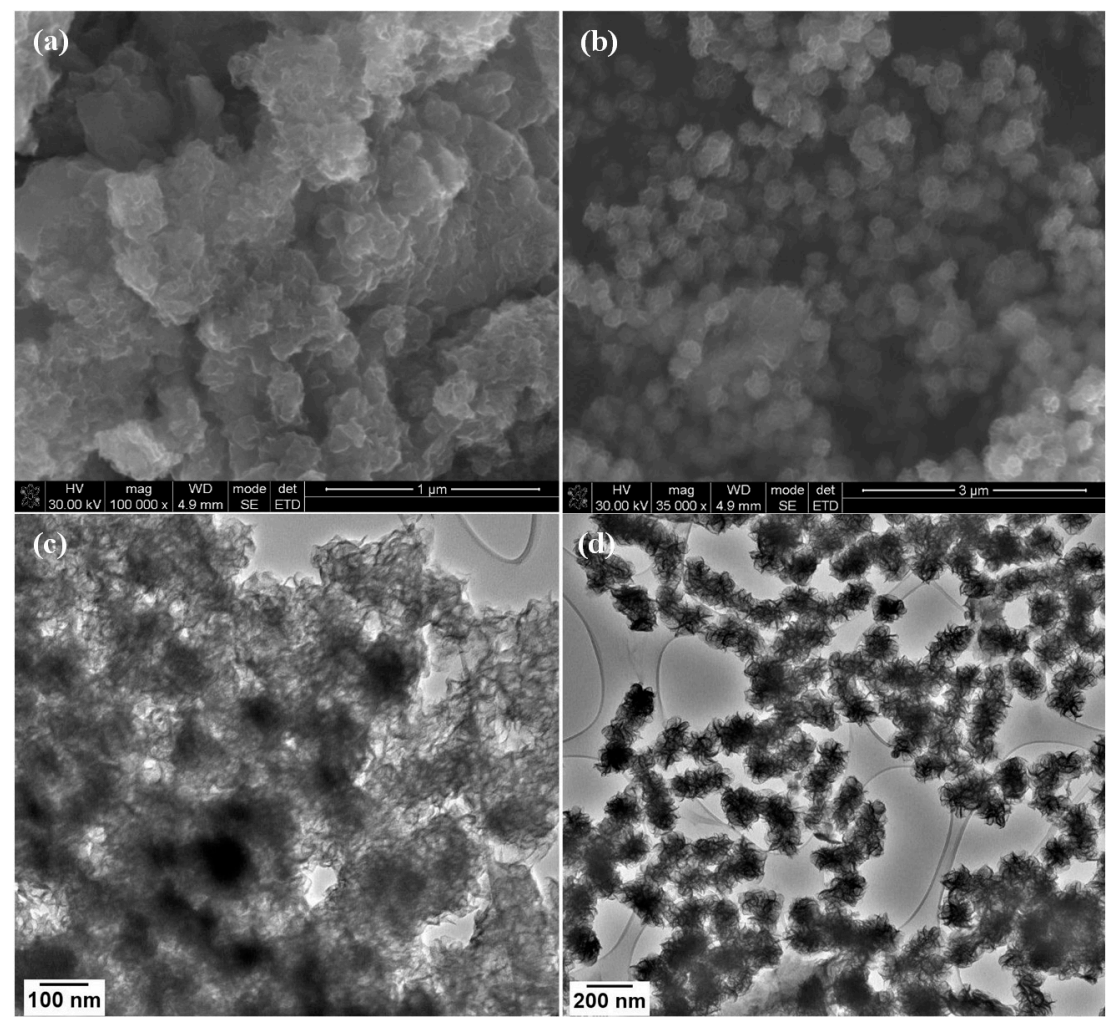

Figure 3. SEM images of (a) $\mathrm{MoSe}_{2}$ nanosheets and (b) $\mathrm{MoSe}_{2}$ nanoflowers and TEM images of (c) $\mathrm{MoSe}_{2}$ nanosheets and (d) $\mathrm{MoSe}_{2}$ nanoflowers.

Figure $4 \mathrm{~d}$ shows that there is a transition that occurs between 30 and 60 min that results in a change of the morphology of the nanosheets. At $30 \mathrm{~min}$ flocculates of the material with small ultrathin nanosheets in an OAm matrix can be observed. As the reaction continues to run for a longer period the flocculate is gradually phased out to form a network of densely packed wrinkled few-layer nanosheets beginning at $60 \mathrm{~min}$ as shown in Figure $4 \mathrm{~d}$. This transition continues from 60 to $120 \mathrm{~min}$ as shown in Figure 4e,f. This transition suggests that the reaction had not run to completion at $30 \mathrm{~min}$, this could be due to the slow release of the Mo monomer as the $\mathrm{Mo}(\mathrm{CO})_{6}$ decomposes via decarbonylation of the $\mathrm{CO}$ ligands [28]. The formation of $\mathrm{MoSe}_{2}$ via $\mathrm{Mo}(\mathrm{CO})_{6}$ precursor is shown in Equations (1) and (2):

$$
\begin{gathered}
2 \mathrm{CH}_{4} \mathrm{~N}_{2} \mathrm{Se} \rightarrow 2 \mathrm{H}_{2} \mathrm{Se}+2 \mathrm{C}(\mathrm{NH})_{2} \\
\mathrm{Mo}(\mathrm{CO})_{6}+2 \mathrm{H}_{2} \mathrm{Se} \rightarrow \mathrm{MoSe}_{2}+6 \mathrm{CO}+2 \mathrm{H}_{2}
\end{gathered}
$$



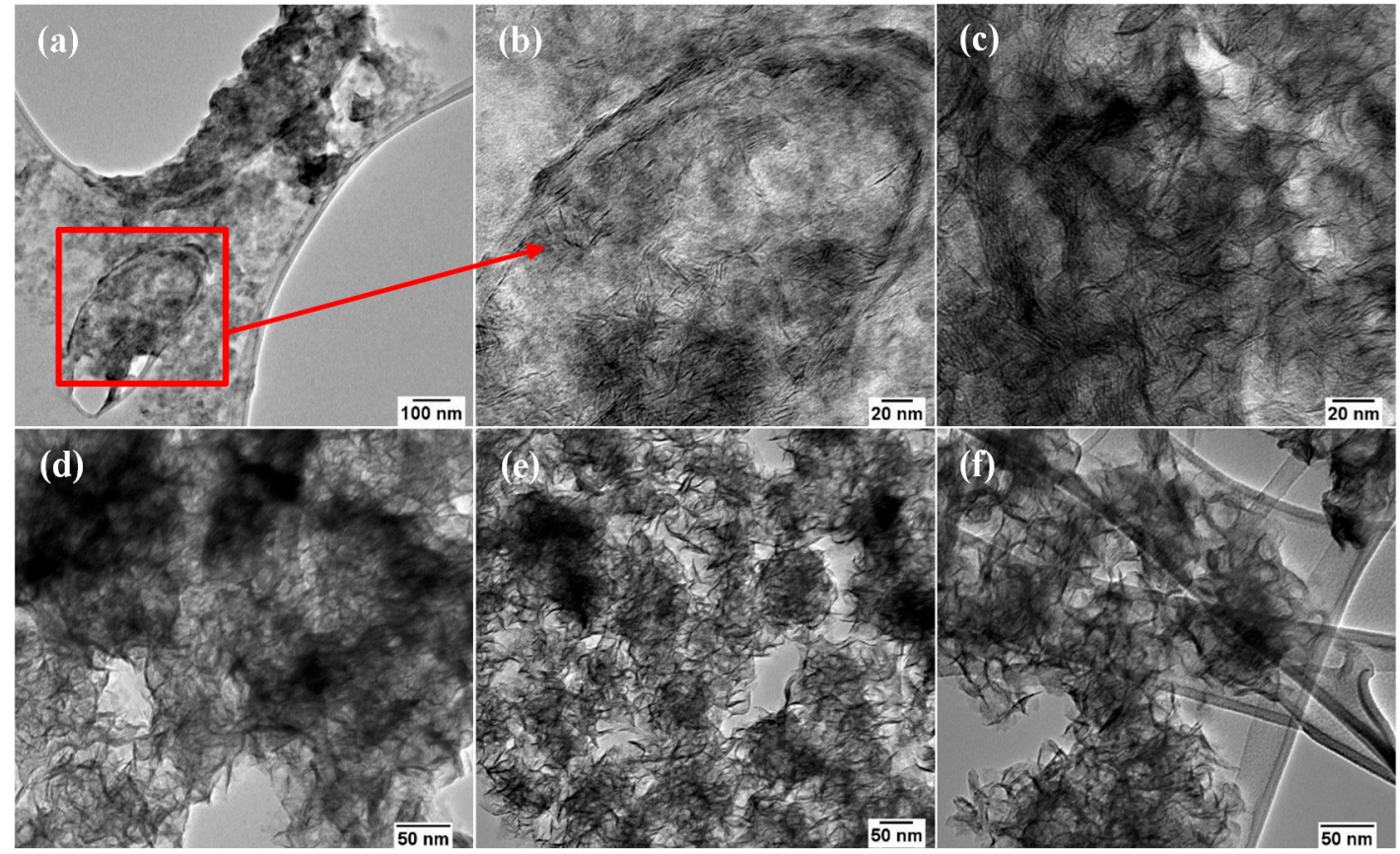

Figure 4. The TEM images of the $\mathrm{MoSe}_{2}$ nanosheets: $(\mathbf{a}-\mathbf{c})$ are images of the nanomaterial taken at $30 \mathrm{~min},(\mathbf{d}-\mathbf{f})$ are the TEM images for MoSe 2 wrinkled few-layer nanosheets at 60, 90 and $120 \mathrm{~min}$.

The amorphous flocculate which is made up of the monomer from the decomposed precursors is consumed as the reaction runs for longer time periods until the large area wrinkled few-layer nanosheets are formed; a schematic diagram of the process is depicted in Scheme 1.

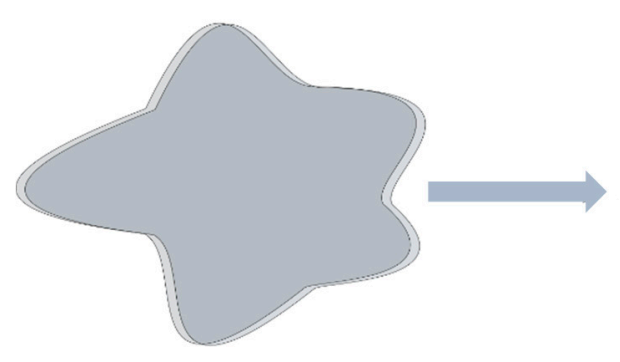

Flocculate

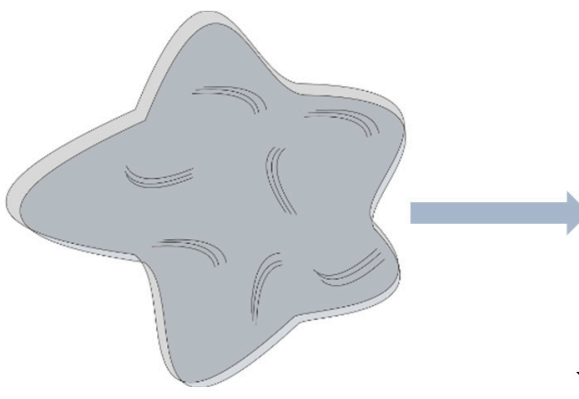

Growing

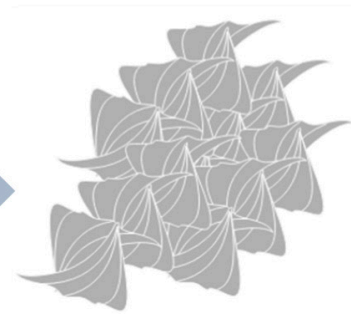

Wrinkled few-layer nanosheet

Scheme 1. Mechanism for the formation of few-layer nanosheets.

This phenomenon was also observed by Gao and co-workers [29], who were able to form few layer nanosheets from the flocculate by increasing the reaction temperature in their microwave synthesis reaction when synthesizing $\mathrm{MoS}_{2}$ nanosheets. They suggested that the few layer nanosheets are formed by consuming the amorphous material in the flocculates as suggested in the process described in this synthetic method. However, in this work it has been proven that the few layer nanosheets form and grow from the flocculates at $300{ }^{\circ} \mathrm{C}$ just by increasing the reaction time from 30 to $60 \mathrm{~min}$, which suggests that the temperature at which the reaction occurs is suitable for the synthesis of the material but longer reaction times are needed for the reaction to run to completion.

To elucidate the formation mechanism for the nanoflowers a time study was also done. However, the nanoflowers had already formed $30 \mathrm{~min}$ into the reaction hence only the $30 \mathrm{~min}$ sample is shown. The TEM and SEM images are shown in Figure $5 a, b$, respectively. The synthesis procedure for $\mathrm{MoSe}_{2}$ nanoflowers was slightly modified from the method used to synthesize the 
wrinkled few-layer nanosheets by changing the metal precursor from molybdenum hexacarbonyl to molybdic acid. It was found that the use of molybdic acid $\left(\mathrm{H}_{2} \mathrm{MoO}_{4}\right)$ resulted in the formation of a network of nanoflower-like nanomaterials that arise through the formation of individual nanoflowers that are formed out of a central core as opposed to the flocculate observed when molybdenum hexacarbonyl was used [30]. Nanosheets with small lateral dimensions grow randomly outward from this central core in a fashion closely resembling a blooming flower. However, the nanoflowers that form do come together and agglomerated forming an interconnected network of these nanoflower structures. A schematic representation of the process is shown in Scheme 2.

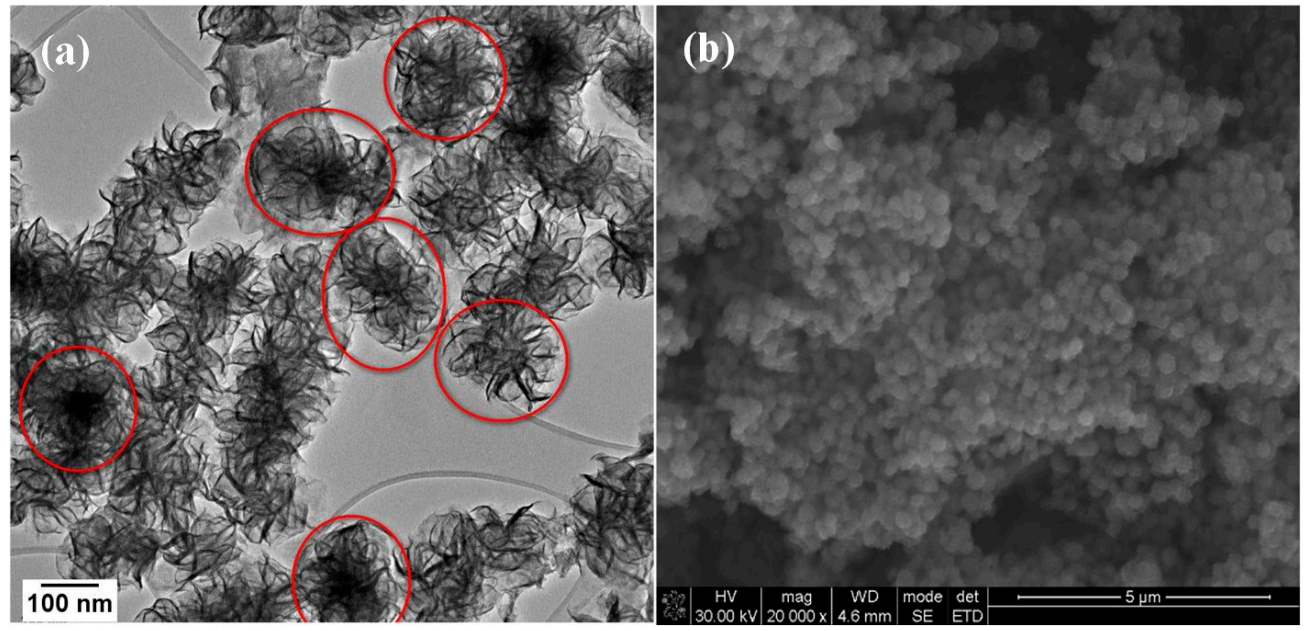

Figure 5. (a) TEM and (b) SEM images of $\mathrm{MoSe}_{2}$ nanoflowers formed at 30 min reaction time.

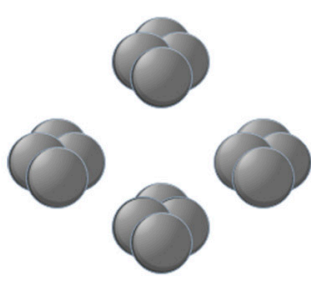

Nucleation of nanoparticles forming dense central core
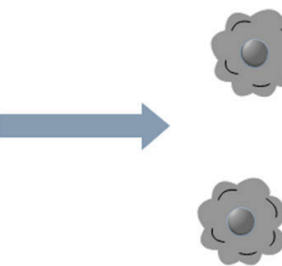

\section{Formation of nanoflowers from} dense central core

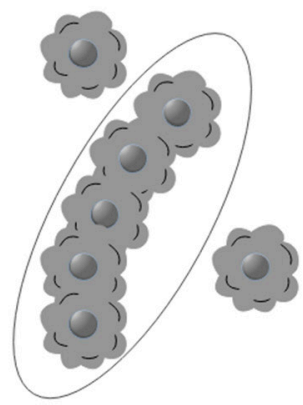

Nanoflowers aggregates to form a network

Scheme 2. Mechanism for the formation of $\mathrm{MoSe}_{2}$ nanoflowers.

The mechanism by which the dense central cores form can be understood by considering the decomposition of molybdic acid in oleylamine. The product obtained when molybdic acid is heated to $300{ }^{\circ} \mathrm{C}$ in oleylamine was found to be a mixture of $\mathrm{MoO}_{4}{ }^{2-}$ and oleylamine; this was confirmed by FTIR shown in Figure S3 [30]. The FTIR spectra show the characteristic antisymmetric stretching vibration of Mo-O-Mo in $\mathrm{MoO}_{4}{ }^{2-}$ at $827 \mathrm{~cm}^{-1}$ [31]. The anion has been shown to react with $\mathrm{S}^{2-}$ in the synthesis of $\mathrm{MoS}_{2}$ to form the intermediate $\mathrm{MoO}_{4}{ }^{2-}$ which converts to the $\mathrm{MoS}_{2}$ as the Mo(VI) is reduced to $\mathrm{Mo}(\mathrm{IV})[32,33]$. A similar phenomenon occurs in the formation of $\mathrm{MoSe}_{2}$. The $\mathrm{MoO}_{4}{ }^{2-}$ has been shown in this work to readily react with $\mathrm{H}_{2} \mathrm{Se}$ to form nanoflowers through the formation of a central nucleation core. This rapid formation of the central core then results in the formation of the nanoflowers with the nanosheets growing outwardly simulating the behaviour of a blooming flower. 
Following the production of $\mathrm{H}_{2} \mathrm{Se}$ from selenourea (Equation (1)), its reaction with $\mathrm{MoO}_{4}{ }^{2-}$ is shown in Equation (3):

$$
\mathrm{MoO}_{4}^{2-}+4 \mathrm{H}^{+}+2 \mathrm{H}_{2} \mathrm{Se} \rightarrow \mathrm{MoSe}_{2}+4 \mathrm{H}_{2} \mathrm{O}
$$

Brunauer-Emmett-Teller (BET) surface area analysis was used to determine the surface area of the $\mathrm{MoSe}_{2}$ wrinkled few-layer nanosheets and the nanoflowers. The surface area of the $\mathrm{MoSe}_{2}$ nanosheets was determined to be $14.1 \mathrm{~m}^{2} / \mathrm{g}$ while the surface area of the $\mathrm{MoSe}_{2}$ nanoflowers was determined to be $36.4 \mathrm{~m}^{2} / \mathrm{g}$. The surface area of the $\mathrm{MoSe}_{2}$ nanoflowers was found to be larger than that of the $\mathrm{MoSe}_{2}$-nanosheets. This was expected because nanoflower structures have been known to possess higher surface area and also be microporous in nature [34].

\subsection{Electrochemical Characterization}

Electrochemical characterization was done on the $\mathrm{MoSe}_{2}$ nanomaterials. This was done in order to show how the control of the morphology using the metal precursors can be used to improve the electrocatalytic activity of $\mathrm{MoSe}_{2}$ nanomaterials towards the hydrogen evolution reaction. Polarisation curves of the $\mathrm{MoSe}_{2}$ nanosheets and $\mathrm{MoSe}_{2}$ nanoflowers are shown in Figure 6a along with the polarization curves for $20 \% \mathrm{Pt} / \mathrm{C}$ and glassy carbon (GC). The $20 \% \mathrm{Pt} / \mathrm{C}$ was used as a standard and the GC polarization curve was done to ensure that the current response from the $\mathrm{MoSe}_{2}$ nanomaterials was not due to the effect of the GC.
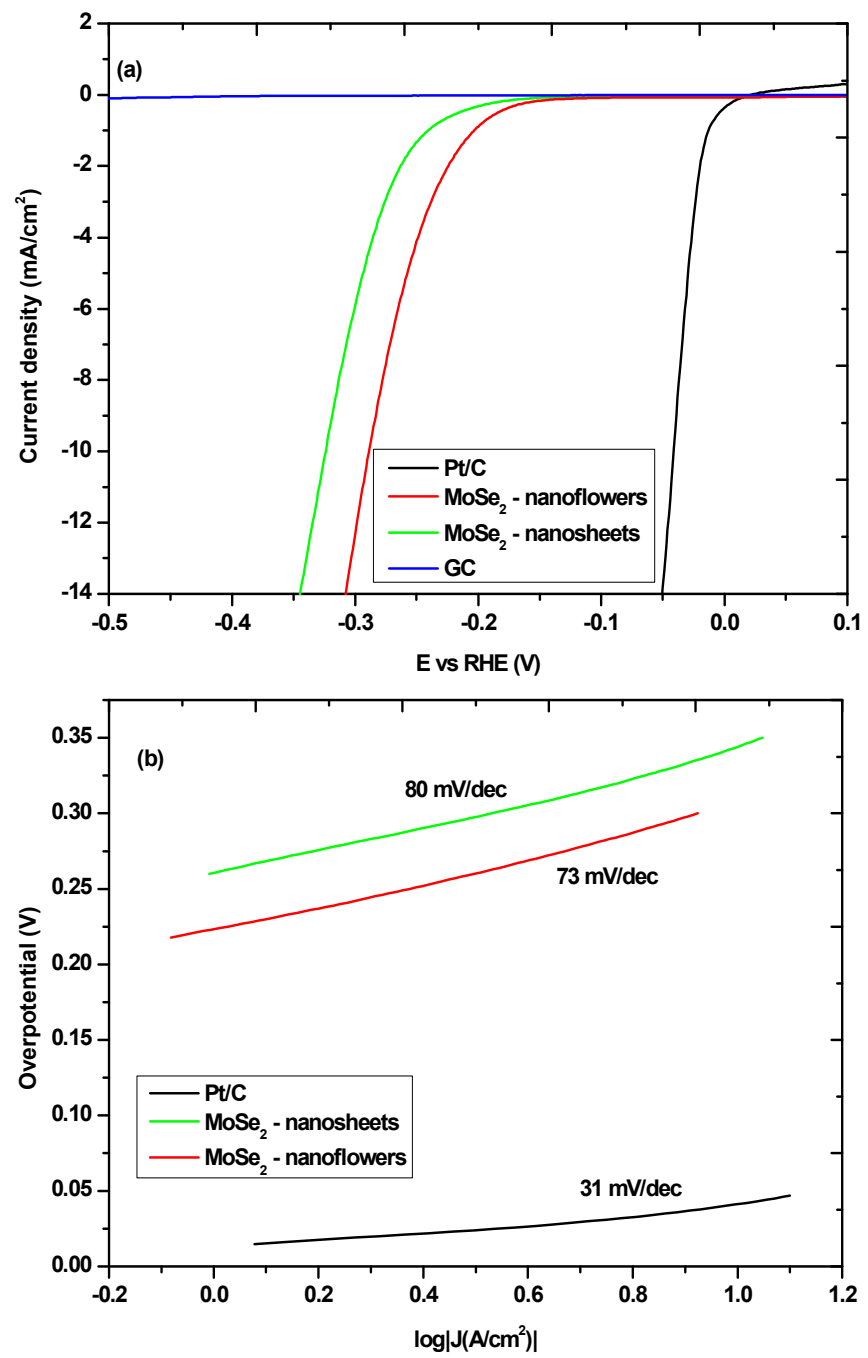

Figure 6. (a) Polarization curves for $\mathrm{MoSe}_{2}$ nanosheets and $\mathrm{MoSe}_{2}$ nanoflowers (b) Tafel slopes for $\mathrm{MoSe}_{2}$ nanosheets and $\mathrm{MoSe}_{2}$ nanoflowers. 
The polarization curves show that the nanoflowers have superior electrocatalytic activity towards the HER compared to nanosheets. The on-set potential for the -nanoflowers was lower than that of the nanosheets at $156 \mathrm{mV}$ and $182 \mathrm{mV}$, respectively. This shows that a smaller overpotential is needed to start the reaction for the nanoflowers than the nanosheets. This trend is also observed when looking at the over-potential needed to reach a specified current density; the over-potentials at approximately $10 \mathrm{~mA} / \mathrm{cm}^{2}\left(\eta_{10}\right)$ for the nanoflowers and nanosheets were found to be $301 \mathrm{mV}$ and $340 \mathrm{mV}$ respectively. As expected, the $\mathrm{Pt} / \mathrm{C}$ sample displayed a lower on-set potential and potential at $10 \mathrm{~mA} / \mathrm{cm}^{2}$. The GC electrode displayed negligible activity towards the HER. The Tafel plots of the MoSe 2 nanomaterials and the $\mathrm{Pt} / \mathrm{C}$ samples are shown in Figure $6 \mathrm{~b}$.

The Tafel plot was used to ascertain the Tafel slope which is a measure of the amount of over-potential needed to increase the reaction rate by a factor of 10 . More accurately the Tafel slope is used to analyze the sensitivity of the current response to the applied potential. The Tafel slope for $\mathrm{Pt} / \mathrm{C}$ was found to be $31 \mathrm{mV} / \mathrm{dec}$ which is roughly the number reported for this particular catalyst [35,36]. The Tafel slope for the $\mathrm{MoSe}_{2}$ nanoflowers was determined to be lower than that of the $\mathrm{MoSe}_{2}$ nanosheets at $73 \mathrm{mV} / \mathrm{dec}$ and $80 \mathrm{mV} / \mathrm{dec}$ respectively. This result shows that the activity of the MoSe nanomaterials is partly dependant on the morphology of the nanomaterials. This result is not surprising as it has been determined that the active sites for the HER in TMDs such as $\mathrm{MoSe}_{2}$ are the edge sites and as such the morphology that can effectively expose these edge sites would, therefore, have a better activity towards the HER. In this case the nanoflowers seem to be more effective at exposing these edge sites compared to the nanosheets as seen by the superior performance of the nanoflowers compared to the nanosheets. This is consistent with what has been reported in the literature with respect to how the nanoflowers morphology seems to be more ideal for the HER compared to other nanomaterial morphologies [36,37]. This can also be attributed to the higher surface area of the nanoflowers compared to the nanosheets which may afford the nanoflowers more available active sites.

To get a better understanding of the role of the surface area on the activity of the catalysts the $C_{d l}$ plots for the two morphologies were obtained in order to get an estimation of the effective electrochemical surface area. Cyclic voltammetry (CV) was employed in order to determine the Cdl, the current response from the CV curves in the potential range between $0.2 \mathrm{~V}$ to $0.6 \mathrm{~V}$ shown in Figure $\mathrm{S} 4$ is due to the double layer charging. The electrochemical double layer can be used to estimate the effective electrochemical surface area because it is expected that the double layer capacitance is linearly proportional to the active surface area [38]. The estimation is done by plotting the difference between anodic and cathodic current densities $\left(\Delta \mathrm{J}=\mathrm{J}_{\mathrm{a}}-\mathrm{J}_{\mathrm{c}}\right)$ vs the scan rate at $0.41 \mathrm{~V}$ vs RHE which is shown in Figure 7a. In this linear plot, the slope is the $C_{\mathrm{dl}}$. As expected, the $\mathrm{C}_{\mathrm{dl}}$ of the MoSe $\mathrm{M}$-nanoflowers was found to be higher than that of the $\mathrm{MoSe}_{2}$ nanosheets which indicates that the nanoflowers have more exposed edge sites. This would then explain why the nanoflowers seem to possess better electrocatalytic performance compared to the nanosheets.

The $\mathrm{C}_{\mathrm{dl}}$ can be used to estimate the electrochemical surface area (ECSA); the ECSA is estimated using the ratio of the $C_{d l}$ to the specific capacitance for the atomically smooth MoSe 2 materials. By assuming that the specific capacitance for the atomically smooth $\mathrm{MoSe}_{2}$ is similar to that of $\mathrm{MoS}_{2}$ and using the Equation (4) [39].

$$
\mathrm{ECSA}=\frac{\mathrm{Cdl}\left(\mathrm{mF} / \mathrm{cm}^{2}\right)}{\operatorname{Cdl}\left(\mu \mathrm{F} / \mathrm{cm}^{2}\right)}
$$

The ECSA of the nanoflowers and nanosheets was found to be 48 and 23, respectively. The higher ECSA of the nanoflowers further shows why the nanoflowers have superior electrochemical properties towards the HER. It should be noted that the wrinkled few-layer nanosheets also have a significant exposure of active edge sites because the nanosheets have a lot of standing edges but the nanoflowers have an advantage because all of the nanosheets that grow from the dense central-core terminate in the active edge sites. The electrode kinetics under HER conditions was investigated using impedance spectroscopy (EIS). The impedance spectra in Figure $7 \mathrm{~b}$ can reveal information about the charge transfer resistance in the Nyquist plot. A small semicircle indicates a small charge transfer resistance which 
is the resistance associated with the transfer of electrons from the electrode material to the ions in solution. When the impedance data was fitted to the appropriate equivalent circuit shown in Figure 8 , the values of the charge transfer resistance $\left(R_{c t}\right)$ were ascertained.
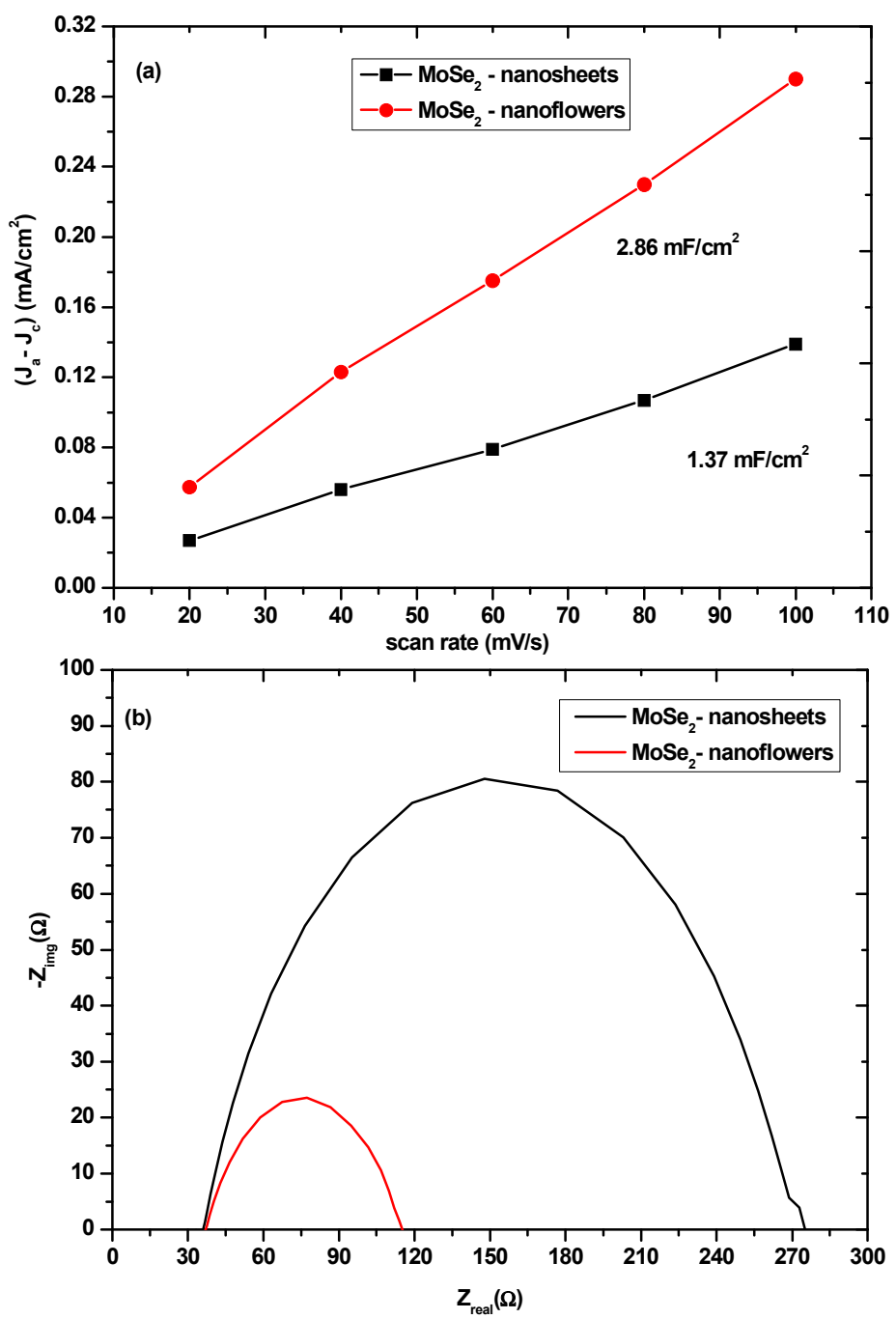

Figure 7. (a) linear fitting of the capacitive currents of $\mathrm{MoSe}_{2}$-nanosheets and $\mathrm{MoSe}_{2}$-nanoflowers vs the increasing scan rates. (b) Nyquist plots of the $\mathrm{MoSe}_{2}$-nanosheets and $\mathrm{MoSe}_{2}$-nanoflowers at $-200 \mathrm{mV}$ vs RHE.

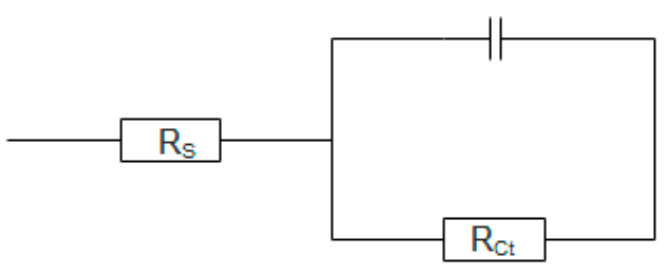

Figure 8. Equivalent circuit of the measured impedance spectra.

The impedance spectra show that the charge transfer resistance of the $\mathrm{MoSe}_{2}$ nanoflowers $(89 \Omega)$ is much smaller than that of the $\mathrm{MoSe}_{2}$ nanosheets $(220 \Omega)$. This suggests that the electron transfer between the electrode material and the electrolyte in the nanoflowers is much more effective than in the nanosheets. Stability studies were also performed on the nanomaterials to determine whether they are stable in acidic media. An initial LSV scan was compared to a LSV scan that was measured 
after a thousand cycles were performed at $100 \mathrm{mV} / \mathrm{s}$. As shown in Figure S5 there was no significant change in the LSV curves after a thousand cycles for both the nanoflowers and the nanosheets which suggest that the nanomaterials are fairly stable in acidic media. A comparison of the overpotential at $10 \mathrm{~mA} / \mathrm{cm}^{2}$ and the Tafel slopes of $\mathrm{MoSe}_{2}$ synthesized using different techniques is shown in Table 1. The electrochemical characterisation for the data in the table was done at $0.5 \mathrm{M} \mathrm{H}_{2} \mathrm{SO}_{4}$. Table 1 shows that the overpotential and Tafel slope of the $\mathrm{MoSe}_{2}$ nanomaterials synthesized using the colloidal synthesis is comparable to those of other synthetic techniques. This is also true for the results found in this study.

Table 1. Comparison of $\eta_{10}$ and Tafel slopes values of $\mathrm{MoSe}_{2}$ synthesized using different techniques.

\begin{tabular}{ccccc}
\hline HER Catalyst & Synthesis Technique & $\eta_{\mathbf{1 0}}(\mathbf{m V})$ & Tafel Slope $(\mathbf{m V} / \mathbf{d e c})$ & Reference \\
\hline MoSe $_{2}$ /GC & LPE & 340 & 88 & {$[21]$} \\
MoSe $_{2}$-nanoflowers & CVD & 220 & 61 & {$[40]$} \\
MoSe $_{2}$-nanosheets & Hydrothermal & 305 & 69 & {$[41]$} \\
MoSe $_{2}$-nanonetworks & Colloidal & 302 & 109 & {$[42]$} \\
$\mathbf{M o S e}_{2}$-nanoflowers & Colloidal & 233 & 89 & {$[42]$} \\
MoSe $_{2}$-nanosheets & Colloidal & 300 & 98 & {$[43]$} \\
$\mathbf{M o S e}_{2}$-nanosheets & Colloidal & 340 & 80 & This work \\
$\mathbf{M o S e}_{2}$-nanoflowers & Colloidal & 301 & 73 & This work \\
\hline
\end{tabular}

\section{Conclusions}

In summary, a colloidal synthetic method was developed to synthesize a network of wrinkled few-layer nanosheets. The method was also adapted to synthesize $\mathrm{MoSe}_{2}$ nanoflowers by using molybdic acid as the metal precursor. The electrochemical properties of the $\mathrm{MoSe}_{2}$ nanosheets and $\mathrm{MoSe}_{2}$ nanoflowers were used to ascertain which morphology of the nanomaterials was most suitable for use as an electrode material for the HER. The overall electrochemical performance of the $\mathrm{MoSe}_{2}$ nanoflowers was determined to be superior to that of the $\mathrm{MoSe}_{2}$ nanosheets due to their higher effective electrochemical surface area. This indicates that the $\mathrm{MoSe}_{2}$ nanoflowers would be more suitable for use as HER electrode materials.

Supplementary Materials: The following are available online at http://www.mdpi.com/2079-4991/10/9/1786/s1, Figure S1: TGA of selenourea showing the decomposition of the compound to $\mathrm{H}_{2} \mathrm{Se}$ and the carboamide $\left(\mathrm{C}(\mathrm{NH})_{2}\right)$ at $\sim 220^{\circ} \mathrm{C}$, Figure S2: HRTEM images of nanosheets synthesized at $30 \mathrm{~min}$, showing the interlayer spacing of the nanosheets. The d-spacing of the lattice fringes can be used to determine whether the synthesized nanosheets are multi-layer or monolayer nanosheets. The d-spacing of the nanosheets was determined to be $\sim 0.66 \mathrm{~nm}$ which is the d-spacing of the (002) lattice plane in multi-layer nanosheets. This confirms that the nanosheets at $30 \mathrm{~min}$ are indeed multi-layer, Figure S3: The FTIR spectrum of the product obtained when $\mathrm{H}_{2} \mathrm{MoO}_{4}$ is heated in oleylamine at $300{ }^{\circ} \mathrm{C}$, Figure S4: CV curves of (a) MoSe 2 -nanosheets and (b) MoSe 2 -nanoflowers at scan-rates of 20, 40, 60, 80 and $100 \mathrm{mV} / \mathrm{s}$, Figure S5: LSV curves of (a) MoSe2-nanoflowers and (b) MoSe2-nanosheets before and after a 1000 cycles of LSV.

Author Contributions: Z.N. was responsible for investigation, formal analysis and writing the original draft. N.S., S.N., T.K. and O.N. were responsible for formal analysis of some of the data and reviewing and editing the manuscript. P.S., Z.N.T. and E.C.L. were responsible for data curation in some of the techniques used, reviewing and editing the manuscript. S.S.G. and N.M. were responsible for conceptualization, supervision, funding acquisition and reviewing and editing of the manuscript. All authors have read and agreed to the published version of the manuscript.

Funding: This research was funded by the National Research Foundation, South Africa (UID: 115448) and the APC was funded by the University of the Witwatersrand.

Acknowledgments: The authors would like to thank the University of the Witwatersrand, the Wits Microscopy and Microanalysis Unit (MMU), and the National Research Foundation (NRF) for their financial support.

Conflicts of Interest: The authors declare no conflicts of interest. 


\section{References}

1. Memaran, S.; Pradhan, N.R.; Lu, Z.; Rhodes, D.; Ludwig, J.; Zhou, Q.; Ogunsolu, O.; Ajayan, P.M.; Smirnov, D.; Fernández-Domínguez, A.I. Pronounced photovoltaic response from multilayered transition-metal dichalcogenides PN-junctions. Nano Lett. 2015, 15, 7532-7538. [CrossRef]

2. Airo, M.A.; Rodrigues, R.; Gqoba, S.; Ntholeng, N.; Otieno, F.; Moloto, M.J.; Greenshields, M.W.C.C.; Hümmelgen, I.A.; Moloto, N. Colloidal InSe nanostructures: Effect of morphology on their chemical sensitivity to methanol and formaldehyde fumes. Sens. Actuators B 2016, 236, 116-125. [CrossRef]

3. Machogo, L.F.E.; Tetyana, P.; Sithole, R.; Gqoba, S.S.; Phao, N.; Airo, M.; Shumbula, P.M.; Moloto, M.J.; Moloto, N. Unravelling the structural properties of mixed-valence $\alpha$-and $\beta$-AuSe nanostructures using XRD, TEM and XPS. Appl. Surf. Sci. 2018, 456, 973-979. [CrossRef]

4. Balasingam, S.K.; Lee, J.S.; Jun, Y. Molybdenum diselenide/reduced graphene oxide based hybrid nanosheets for supercapacitor applications. Dalton Trans. 2016, 45, 9646-9653. [CrossRef]

5. Jeong, Y.; Park, J.H.; Ahn, J.; Lim, J.Y.; Im, S. 2D MoSe 2 field-effect transistor with small threshold voltage for piezoelectric touch sensor applications. ECS Meet. Abstr. 2018, MA2018-01, 2624.

6. Ge, J.; Fan, L.; Wang, J.; Zhang, Q.; Liu, Z.; Zhang, E.; Liu, Q.; Yu, X.; Lu, B. MoSe 2 /N-doped carbon as anodes for potassium-ion batteries. Adv. Energy Mater. 2018, 8, 1801477. [CrossRef]

7. Mao, B.; Bao, T.; Yu, J.; Zheng, L.; Qin, J.; Yin, W.; Cao, M. One-pot synthesis of MoSe 2 hetero-dimensional hybrid self-assembled by nanodots and nanosheets for electrocatalytic hydrogen evolution and photothermal therapy. Nano Res. 2017, 10, 2667-2682. [CrossRef]

8. Zhang, C.; Chen, X.; Peng, Z.; Fu, X.; Lian, L.; Luo, W.; Zhang, J.; Li, H.; Wang, Y.; Zhang, D. Phosphine-free synthesis and shape evolution of $\mathrm{MoSe}_{2}$ nanoflowers for electrocatalytic hydrogen evolution reactions. CrystEngComm 2018, 20, 2491-2498. [CrossRef]

9. Yang, H.; Zhao, J.; Wu, C.; Ye, C.; Zou, D.; Wang, S. Facile synthesis of colloidal stable MoS $_{2}$ nanoparticles for combined tumor therapy. Chem. Eng. J. 2018, 351, 548-558. [CrossRef]

10. Sun, Y.; Alimohammadi, F.; Zhang, D.; Guo, G. Enabling colloidal synthesis of edge-oriented $\mathrm{MoS}_{2}$ with expanded interlayer spacing for enhanced HER catalysis. Nano Lett. 2017, 17, 1963-1969. [CrossRef]

11. Lin, H.; Wang, C.; Wu, J.; Xu, Z.; Huang, Y.; Zhang, C. Colloidal synthesis of $\mathrm{MoS}_{2}$ quantum dots: Size-dependent tunable photoluminescence and bioimaging. New J. Chem. 2015, 39, 8492-8497. [CrossRef]

12. van der Stam, W.; Akkerman, Q.A.; Ke, X.; van Huis, M.A.; Bals, S.; de Mello Donega, C. Solution-Processable Ultrathin Size-and Shape-Controlled Colloidal $\mathrm{Cu}_{2}-\mathrm{x}$ S Nanosheets. Chem. Mater. 2014, 27, $283-291$. [CrossRef]

13. Geisenhoff, J.Q.; Tamura, A.K.; Schimpf, A. Using ligands to control reactivity, size and phase in the colloidal synthesis of $\mathrm{WSe}_{2}$ nanocrystals. Chem. Commun. 2019, 55, 8856-8859. [CrossRef]

14. Mansouri, A.; Semagina, N. Colloidal synthesis protocol of shape-and dimensionally-controlled transition-metal chalcogenides and their hydrodesulfurization activities. ACS Appl. Nano Mater. 2018, 1, 4408-4412. [CrossRef]

15. Yoo, D.; Kim, M.; Jeong, S.; Han, J.; Cheon, J. Chemical synthetic strategy for single-layer transition-metal chalcogenides. J. Am. Chem. Soc. 2014, 136, 14670-14673. [CrossRef]

16. Sayed, F.N.; Polshettiwar, V. Facile and sustainable synthesis of shaped iron oxide nanoparticles: Effect of iron precursor salts on the shapes of iron oxides. Sci. Rep. 2015, 5, 1-14. [CrossRef]

17. Jangir, L.K.; Kumari, Y.; Kumar, A.; Kumar, M.; Awasthi, K. Investigation of luminescence and structural properties of $\mathrm{ZnO}$ nanoparticles, synthesized with different precursors. Mater. Chem. Front. 2017, 1, 1413-1421. [CrossRef]

18. Arellano, J.S.; Rosendo, E.; Romano, R.; Nieto, G.; Diaz, T.; García, G.; Juárez, H.; Pacio, M.; Galeazzi, R.; Morales, C. Synthesis and characterization of CdSe nanoparticles with cadmium precursor variation in colloidal synthesis. Adv. Mate. Res. 2014, 976, 52-58. [CrossRef]

19. Ghritlahre, V.; Kumari, J.; Agarwal, P. Synthesis and study of molybdenum diselenide (MoSe2) by Solvo-thermal method. AIP Conf. Proc. 2018, 1953, 050048.

20. Mazloomi, K.; Gomes, C. Hydrogen as an energy carrier: Prospects and challenges. Renew. Sustain. Energy Rev. 2012, 16, 3024-3033. [CrossRef] 
21. Najafi, L.; Bellani, S.; Oropesa-Nuñez, R.; Ansaldo, A.; Prato, M.; Del Rio Castillo, A.E.; Bonaccorso, F. Engineered $\mathrm{MoSe}_{2}$-based heterostructures for efficient electrochemical hydrogen evolution reaction. Adv. Energy Mater. 2018, 8, 1703212. [CrossRef]

22. Wang, H.; Kong, D.; Johanes, P.; Cha, J.J.; Zheng, G.; Yan, K.; Liu, N.; Cui, Y. MoSe 2 and WSe 2 nanofilms with vertically aligned molecular layers on curved and rough surfaces. Nano Lett. 2013, 13, 3426-3433. [CrossRef] [PubMed]

23. Kong, D.; Wang, H.; Cha, J.J.; Pasta, M.; Koski, K.J.; Yao, J.; Cui, Y. Synthesis of MoS 2 and MoSe 2 films with vertically aligned layers. Nano Lett. 2013, 13, 1341-1347. [CrossRef] [PubMed]

24. Joensen, P.; Frindt, R.; Morrison, S.R. Single-layer MoS 2 . Mater. Res. Bull. 1986, 21, 457-461. [CrossRef]

25. Tonndorf, P.; Schmidt, R.; Böttger, P.; Zhang, X.; Börner, J.; Liebig, A.; Albrecht, M.; Kloc, C.; Gordan, O.; Zahn, D.R. Photoluminescence emission and Raman response of monolayer $\mathrm{MoS}_{2}, \mathrm{MoSe}_{2}$, and $\mathrm{WSe}_{2}$. Opt. Express 2013, 21, 4908-4916. [CrossRef]

26. Niu, L.; Li, K.; Zhen, H.; Chui, Y.S.; Zhang, W.; Yan, F.; Zheng, Z. Salt-assisted high-throughput synthesis of single-and few-layer transition metal dichalcogenides and their application in organic solar cells. Small 2014, 10, 4651-4657. [CrossRef]

27. Savjani, N.; Lewis, E.A.; Bissett, M.A.; Brent, J.R.; Dryfe, R.A.; Haigh, S.J.; O’Brien, P. Synthesis of lateral size-controlled monolayer $1 \mathrm{H}-\mathrm{MoS}_{2} @$ oleylamine as supercapacitor electrodes. Chem. Mater. 2016, 28, 657-664. [CrossRef]

28. Cho, C.; Bernasek, S. Molybdenum deposition from the decomposition of molybdenum hexacarbonyl. J. Appl. Phys. 1989, 65, 3035-3043. [CrossRef]

29. Gao, M.-R.; Chan, M.K.; Sun, Y. Edge-terminated molybdenum disulfide with a 9.4-Å interlayer spacing for electrochemical hydrogen production. Nat. Commun. 2015, 6, 1-8. [CrossRef]

30. Sun, D.; Feng, S.; Terrones, M.; Schaak, R.E. Formation and interlayer decoupling of colloidal $\mathrm{MoSe}_{2}$ nanoflowers. Chem. Mater. 2015, 27, 3167-3175. [CrossRef]

31. Yu, X.; Wang, J.; Zhang, M.; Yang, P.; Yang, L.; Cao, D.; Li, J. One-step synthesis of lamellar molybdate pillared hydrotalcite and its application for AZ31 Mg alloy protection. Solid State Sci. 2009, 11, 376-381. [CrossRef]

32. Ghosh, S.; Srivastava, C.; Nath, S.; Celis, J.-P. Simple formation of nanostructured molybdenum disulfide thin films by electrodeposition. Int. J. Electrochem. 2013, 2013, 1-8. [CrossRef]

33. Zhang, H. Synthesis of Highly Active Unsupported Molybdenum Sulfide Catalysts for Hydrosulfurization and Hydrodeoxygenation; University of New Brunswick: Bathurst, NB, Canada, 2014.

34. Wang, R.; Jayakumar, A.; Xu, C.; Lee, J.-M. Ni(OH) $)_{2}$ nanoflowers/graphene hydrogels: A new assembly for supercapacitors. ACS Sustain. Chem. Eng. 2016, 4, 3736-3742. [CrossRef]

35. Zhao, W.; Dong, B.; Guo, Z.; Su, G.; Gao, R.; Wang, W.; Cao, L. Colloidal synthesis of VSe 2 single-layer nanosheets as novel electrocatalysts for the hydrogen evolution reaction. Chem. Commun. 2016, 52, 9228-9231. [CrossRef] [PubMed]

36. Qu, Y.; Shao, M.; Shao, Y.; Yang, M.; Xu, J.; Kwok, C.T.; Shi, X.; Lu, Z.; Pan, H. Ultra-high electrocatalytic activity of $\mathrm{VS}_{2}$ nanoflowers for efficient hydrogen evolution reaction. J. Mater. Chem. A 2017, 5, 15080-15086. [CrossRef]

37. Cao, J.; Zhang, X.; Zhang, Y.; Zhou, J.; Chen, Y.; Liu, X. Free $\mathrm{MoS}_{2}$ nanoflowers grown on graphene by microwave-assisted synthesis as highly efficient non-noble-metal electrocatalysts for the hydrogen evolution reaction. PLoS ONE 2016, 11, e0161374. [CrossRef]

38. Qi, F.; Wang, X.; Zheng, B.; Chen, Y.; Yu, B.; Zhou, J.; He, J.; Li, P.; Zhang, W.; Li, Y. Self-assembled chrysanthemum-like microspheres constructed by few-layer $\mathrm{ReSe}_{2}$ nanosheets as a highly efficient and stable electrocatalyst for hydrogen evolution reaction. Electrochim. Acta 2017, 224, 593-599. [CrossRef]

39. Benson, J.; Li, M.; Wang, S.; Wang, P.; Papakonstantinou, P. Electrocatalytic hydrogen evolution reaction on edges of a few layer molybdenum disulfide nanodots. ACS Appl. Mater. Interfaces 2015, 7, 14113-14122. [CrossRef]

40. Masurkar, N.; Thangavel, N.K.; Arava, L.M.R. CVD-grown MoSe 2 nanoflowers with dual active sites for efficient electrochemical hydrogen evolution reaction. ACS Appl. Mater. Interfaces 2018, 10, 27771-27779. [CrossRef]

41. Zhang, L.; Wang, T.; Sun, L.; Sun, Y.; Hu, T.; Xu, K.; Ma, F. Hydrothermal synthesis of 3D hierarchical $\mathrm{MoSe}_{2} / \mathrm{NiSe}_{2}$ composite nanowires on carbon fiber paper and their enhanced electrocatalytic activity for the hydrogen evolution reaction. J. Mater. Chem. A 2017, 5, 19752-19759. [CrossRef] 
42. Guo, W.; Chen, Y.; Wang, L.; Xu, J.; Zeng, D.; Peng, D.-L. Colloidal synthesis of MoSe 2 nanonetworks and nanoflowers with efficient electrocatalytic hydrogen-evolution activity. Electrochim. Acta 2017, 231, 69-76. [CrossRef]

43. Zhou, X.; Jiang, J.; Ding, T.; Zhang, J.; Pan, B.; Zuo, J.; Yang, Q. Fast colloidal synthesis of scalable Mo-rich hierarchical ultrathin $\mathrm{MoSe}_{2-\mathrm{x}}$ nanosheets for high-performance hydrogen evolution. Nanoscale 2014, 6, 11046-11051. [CrossRef] [PubMed] 